\title{
THE NUMERICAL SOLUTION OF THE TRANSIENT HEAT CONDUCTION PROBLEM USING THE LATTICE BOLTZMANN METHOD
}

\author{
Grażyna Katuża \\ Silesian University of Technology, Poland \\ grazyna.kaluza@polsl.pl
}

\begin{abstract}
The implementation of the lattice Boltzmann method (LBM) for the solution of the transient heat conduction problem is presented. The one dimensional task is considered and the different boundary conditions, specifically the Dirichlet, Neumann and Robin ones are taken into account. The D1Q2 lattice model is applied. To check the accuracy of the LBM algorithm, the same problems have been solved using the explicit variant of the finite difference method. In the final part of the paper, the results of computations are shown and the conclusions are formulated.
\end{abstract}

\section{Introduction}

Over the last decade the lattice Boltzmann method (LBM) has been developed as a promising computational tool to analyze the large class of engineering problems, among others, the heat transfer problems $[1,2]$. In this paper the LBM has been applied in order to solve the Fourier equation

$$
X \in D: \quad c \frac{\partial T(X, t)}{\partial t}=\lambda \nabla^{2} T(X, t)+Q(X, t)
$$

where $\lambda$ is the thermal conductivity, $c$ is the volumetric specific heat, $Q(X, t)$ is the source function, $T, X, t$ denote the temperature, spatial co-ordinates and time. The equation (1) is supplemented by boundary conditions

$$
\begin{array}{ll}
X \in \Gamma_{1}: & T(X, t)=T_{b} \\
X \in \Gamma_{2}: & q(X, t)=-\lambda \frac{\partial T(X, t)}{\partial n}=q_{b} \\
X \in \Gamma_{3}: & q(X, t)=-\lambda \frac{\partial T(X, t)}{\partial n}=\alpha\left[T(X, t)-T_{a}\right]
\end{array}
$$


and the initial one

$$
t=0: T(X, 0)=T_{p}
$$

where $\partial T / \partial n$ denotes the normal derivative, $n$ is the normal outward vector, $T_{b}, q_{b}$ are the known boundary temperature and boundary heat flux, respectively, $\alpha$ is the heat transfer coefficient, $T_{a}$ is the ambient temperature and $T_{p}$ is the initial temperature.

\section{Kinetic equation}

The Boltzmann transport equation can be written as [2]

$$
\frac{\partial f}{\partial t}+\mathbf{e} \cdot \nabla f=\Omega
$$

where $f$ is the distribution function, $\mathbf{e}$ is the velocity and $\Omega$ is the collision operator. It should be pointed out that it is difficult to solve equation (4) because $\Omega$ is a function of $f$ and in a general case it is an integro-differential equation [2].

The starting point of the lattice Boltzmann method (LBM) is the kinetic equation $[1,3,4]$

$$
\frac{\partial f_{i}(X, t)}{\partial t}+e_{i} \cdot \nabla f_{i}(X, t)=\Omega_{i}, \quad i=1,2,3, \ldots M
$$

where $f_{i}$ is the particle distribution function denoting the number of particles at the lattice node $x$ at the time $t$ moving in direction $i$ with the velocity $e_{i}$ along the lattice link $h=e_{i} \Delta t$ connecting the nearest neighbors and $M$ is the number of directions in a lattice through which the information propagates. The term $\Omega_{i}$ represents the rate of change of $f_{i}$ due to collisions and is very complicated [2]. $[1,4]$

The simplest model for $\Omega_{i}$ is the Bhatnagar-Gross-Krook (BGK) approximation

$$
\Omega_{i}=-\frac{1}{\tau}\left[f_{i}(X, t)-f_{i}^{0}(X, t)\right]
$$

where $\tau$ is the relaxation time and $f_{i}^{0}(X, t)$ is the equilibrium distribution function.

It should be pointed out that in the case of heat transfer problems the equilibrium distribution function is given by

$$
f_{i}^{0}(X, t)=w_{i} T(X, t)
$$

where $w_{i}$ are the known weights, at the same time $\sum_{i=1}^{M} w_{i}=1$. 
Additionally, the temperature $T$ at the lattice node $x$ and for time $t$ is calculated using the formula $[1,3]$

$$
T(X, t)=\sum_{i=1}^{M} f_{i}(X, t)
$$

From equations (7), (8) results that

$$
\sum_{i=1}^{M} f_{i}^{0}(X, t)=\sum_{i=1}^{M} w_{i} T(X, t)=T(X, t)=\sum_{i=1}^{M} f_{i}(X, t)
$$

\section{Lattice Boltzmann method for 1D problem}

In this paper, for 1D problem, D1Q2 lattice model [1, 5] has been used as shown in Figure 1 . Nodes 0 and $n$ are the boundary ones, while the nodes 1, 2, $\ldots n-1$ are the internal ones.

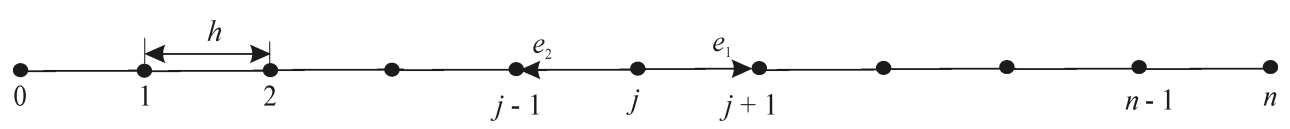

Fig. 1. D1Q2 lattice

In such case the lattice Boltzmann transport equation can be written as [4]

$$
\frac{\partial f_{i}(x, t)}{\partial t}+e_{i} \frac{\partial f_{i}(x, t)}{\partial x}=-\frac{1}{\tau}\left[f_{i}(x, t)-f_{i}^{0}(x, t)\right]+w_{i} \frac{Q(x, t)}{c}, \quad i=1,2
$$

For the D1Q2 lattice, the two velocities $e_{i}$ and their corresponding weights $w_{i}$ are as follows

$$
\begin{aligned}
& e_{1}=v, \quad e_{2}=-v \\
& w_{1}=w_{2}=\frac{1}{2}
\end{aligned}
$$

at the same time $v=h / \Delta t$ ( $h$ is the lattice distance from node to node).

The relaxation time $\tau$ is computed from [1]

$$
\tau=\frac{a}{v^{2}}+\frac{\Delta t}{2}
$$

where $a=\lambda / c$ is the thermal diffusivity and $\Delta t$ is the time step. 
In other words, two equations should be solved, namely

$$
\begin{aligned}
& \frac{\partial f_{1}(x, t)}{\partial t}+v \frac{\partial f_{1}(x, t)}{\partial x}=-\frac{1}{\tau}\left[f_{1}(x, t)-f_{1}^{0}(x, t)\right]+w_{1} \frac{Q(x, t)}{c} \\
& \frac{\partial f_{2}(x, t)}{\partial t}-v \frac{\partial f_{2}(x, t)}{\partial x}=-\frac{1}{\tau}\left[f_{2}(x, t)-f_{2}^{0}(x, t)\right]+w_{2} \frac{Q(x, t)}{c}
\end{aligned}
$$

The approximation of the first derivatives using right-hand side differential quotients is the following $[2,6,7]$

$$
\begin{gathered}
\frac{\partial f_{1}}{\partial t}=\frac{f_{1}(x, t+\Delta t)-f_{1}(x, t)}{\Delta t} \\
\frac{\partial f_{1}}{\partial x}=\frac{f_{1}(x+h, t+\Delta t)-f_{1}(x, t+\Delta t)}{h}
\end{gathered}
$$

and using left-hand side differential quotients is of the form

$$
\begin{gathered}
\frac{\partial f_{2}}{\partial t}=\frac{f_{2}(x, t+\Delta t)-f_{2}(x, t)}{\Delta t} \\
\frac{\partial f_{2}}{\partial x}=\frac{f_{2}(x, t+\Delta t)-f_{2}(x-h, t+\Delta t)}{h}
\end{gathered}
$$

Introducing (15) into (13) and (16) into (14), respectively, one obtains

$$
\begin{gathered}
\frac{f_{1}(x, t+\Delta t)-f_{1}(x, t)}{\Delta t}+v \frac{f_{1}(x+h, t+\Delta t)-f_{1}(x, t+\Delta t)}{h}= \\
=-\frac{1}{\tau}\left[f_{1}(x, t)-f_{1}^{0}(x, t)\right]+w_{1} \frac{Q(x, t)}{c}
\end{gathered}
$$

and

$$
\begin{gathered}
\frac{f_{2}(x, t+\Delta t)-f_{2}(x, t)}{\Delta t}-v \frac{f_{2}(x, t+\Delta t)-f_{2}(x-h, t+\Delta t)}{h}= \\
=-\frac{1}{\tau}\left[f_{2}(x, t)-f_{2}^{0}(x, t)\right]+w_{2} \frac{Q(x, t)}{c}
\end{gathered}
$$

From equations (17) and (18) results that

$$
\begin{aligned}
& f_{1}(x+h, t+\Delta t)=\left(1-\frac{\Delta t}{\tau}\right) f_{1}(x, t)+\frac{\Delta t}{\tau} f_{1}^{0}(x, t)+w_{1} \Delta t \frac{Q(x, t)}{c} \\
& f_{2}(x-h, t+\Delta t)=\left(1-\frac{\Delta t}{\tau}\right) f_{2}(x, t)+\frac{\Delta t}{\tau} f_{2}^{0}(x, t)+w_{2} \Delta t \frac{Q(x, t)}{c}
\end{aligned}
$$


this means

$$
\begin{aligned}
& f_{1 j+1}^{p+1}=\left(1-\frac{\Delta t}{\tau}\right) f_{1 j}^{p}+\frac{\Delta t}{\tau} f_{1 j}^{0 p}+w_{1} \Delta t \frac{Q_{j}^{p}}{c}, \quad j=0,1, \ldots, n-1 \\
& f_{2 j-1}^{p+1}=\left(1-\frac{\Delta t}{\tau}\right) f_{2 j}^{p}+\frac{\Delta t}{\tau} f_{2 j}^{0 p}+w_{2} \Delta t \frac{Q_{j}^{p}}{c}, \quad j=n, n-1, \ldots, 1
\end{aligned}
$$

In equations (20) (c.f. formula (7)):

$$
f_{1 j}^{0 p}=w_{1} T_{j}^{p}, \quad f_{2 j}^{0 p}=w_{2} T_{j}^{p}
$$

where $T_{j}^{p}$ denotes the temperature at the node $j$ and time $t^{p}$.

Additionally

$$
T_{j}^{p+1}=f_{1 j}^{p+1}+f_{2 j}^{p+1}
$$

It should be pointed out that in numerical realization it is convenient to divide the algorithm into two steps:

- collision step: for each node the right-hand sides of equations (20) are calculated

$$
\begin{aligned}
& f_{1 j}^{p+1}=\left(1-\frac{\Delta t}{\tau}\right) f_{1 j}^{p}+\frac{\Delta t}{\tau} f_{1 j}^{0 p}+w_{1} \Delta t \frac{Q_{j}^{p}}{c}, \quad j=0,1,2, \ldots, n \\
& f_{2 j}^{p+1}=\left(1-\frac{\Delta t}{\tau}\right) f_{2 j}^{p}+\frac{\Delta t}{\tau} f_{2 j}^{0 p}+w_{2} \Delta t \frac{Q_{j}^{p}}{c}, \quad j=0,1,2, \ldots, n
\end{aligned}
$$

- streaming step: obtained values are assigned to the adequate nodes (Fig. 2)

$$
\begin{aligned}
& f_{1 j+1}^{p+1}=f_{1 j}^{p+1}, \quad j=n-1, n-2, \ldots, 0 \\
& f_{2 j-1}^{p+1}=f_{2 j}^{p+1}, \quad j=1,2, \ldots, n
\end{aligned}
$$

It is visible that two values are unknown, this means $f_{10}^{p+1}, f_{2 n}^{p+1}$ and these values are determined from the boundary conditions.

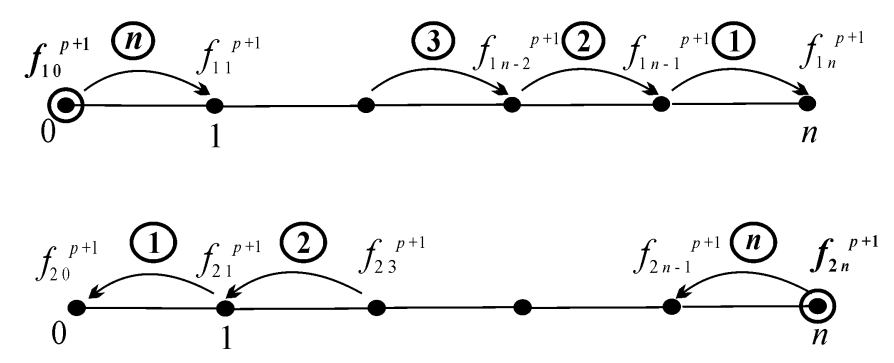

Fig. 2. Streaming process 
For example, if for $x=0$ and for $x=L$ the Dirichlet conditions: $T(x, 0)=T_{w}$, $T(x, L)=T_{z}$ are assumed then (c.f. formula (22))

$$
f_{10}^{p+1}+f_{20}^{p+1}=T_{w} \rightarrow f_{10}^{p+1}=T_{w}-f_{20}^{p+1}
$$

and

$$
f_{1 n}^{p+1}+f_{2 n}^{p+1}=T_{z} \rightarrow f_{2 n}^{p+1}=T_{z}-f_{1 n}^{p+1}
$$

In the case when for $x=L$ the Neumann condition: $-\lambda \partial T(x, t) / \partial x=q_{b}$ is accepted, one has

$$
-\lambda \frac{T_{n}^{p+1}-T_{n-1}^{p+1}}{h}=q_{b} \rightarrow T_{n}^{p+1}=T_{n-1}^{p+1}-\frac{h}{\lambda} q_{b}
$$

and next

$$
f_{1 n}^{p+1}+f_{2 n}^{p+1}=f_{1 n-1}^{p+1}+f_{2 n-1}^{p+1}-\frac{h}{\lambda} q_{b} \rightarrow f_{2 n}^{p+1}=f_{1 n-1}^{p+1}+f_{2 n-1}^{p+1}-f_{1 n}^{p+1}-\frac{h}{\lambda} q_{b}
$$

For Robin condition: $x=L:-\lambda \partial T(x, t) / \partial x=\alpha\left[T(x, t)-T_{a}\right]$ one has

$$
-\lambda \frac{T_{n}^{p+1}-T_{n-1}^{p+1}}{h}=\alpha\left(T_{n}^{p+1}-T_{a}\right) \rightarrow T_{n}^{p+1}=\frac{\lambda}{\lambda+\alpha h} T_{n-1}^{p+1}+\frac{\alpha h}{\lambda+\alpha h} T_{a}
$$

this means

$$
\begin{gathered}
f_{1 n}^{p+1}+f_{2 n}^{p+1}=\frac{\lambda}{\lambda+\alpha h}\left(f_{1 n-1}^{p+1}+f_{2 n-1}^{p+1}\right)+\frac{\alpha h}{\lambda+\alpha h} T_{a} \rightarrow f_{2 n}^{p+1}= \\
\frac{\lambda}{\lambda+\alpha h}\left(f_{1 n-1}^{p+1}+f_{2 n-1}^{p+1}\right)-f_{1 n}^{p+1}+\frac{\alpha h}{\lambda+\alpha h} T_{a}
\end{gathered}
$$

\section{Finite difference method}

To verify the LBM the same problem has been solved using the explicit scheme of the finite difference method (FDM) [6-8]. For 1D problem and internal node $j$ the following approximation of equation (1) is used

$$
c \frac{T_{j}^{p+1}-T_{j}^{p}}{\Delta t}=\lambda \frac{T_{j-1}^{p}-2 T_{j}^{p}+T_{j+1}^{p}}{h^{2}}+Q_{j}^{p}
$$


where $h$ is the constant mesh step, $T_{j-1}^{p}=T\left(x_{j-1}, t^{p}\right), T_{j}^{p}=T\left(x_{j}, t^{p}\right)$ etc.

From equation (31) results that

$$
T_{j}^{p+1}=\left(1-\frac{2 a \Delta t}{h^{2}}\right) T_{j}^{p}+\frac{a \Delta t}{h^{2}}\left(T_{j-1}^{p}+T_{j+1}^{p}\right)+\frac{Q_{j}^{p}}{c} \Delta t
$$

The criterion of stability of this explicit differential scheme is following: $1-\left(2 a \Delta t / h^{2}\right) \geq 0[7,8]$.

\section{Results of computations}

The layer of thickness $L=0.05 \mathrm{~m}$ made of steel is considered. In computations the following input data are introduced: $\lambda=35 \mathrm{~W} /(\mathrm{mK}), c=4.875 \mathrm{MJ} /\left(\mathrm{m}^{3} \mathrm{~K}\right)$, source function $Q=0$. Initial temperature equals to $T_{p}=0^{\circ} \mathrm{C}$, mesh step: $h=0.00125 \mathrm{~m}(n=40)$, time step: $\Delta t=0.1 \mathrm{~s}$.

In the first example of computations the Dirichlet conditions in the form $T(0, t)=$ $=0^{\circ} \mathrm{C}$ and $T(L, t)=100^{\circ} \mathrm{C}$ are assumed. In Figure 3 the temperature distributions obtained by LBM algorithm (symbols) and by FDM algorithm (solid lines) for different moments of time are shown.

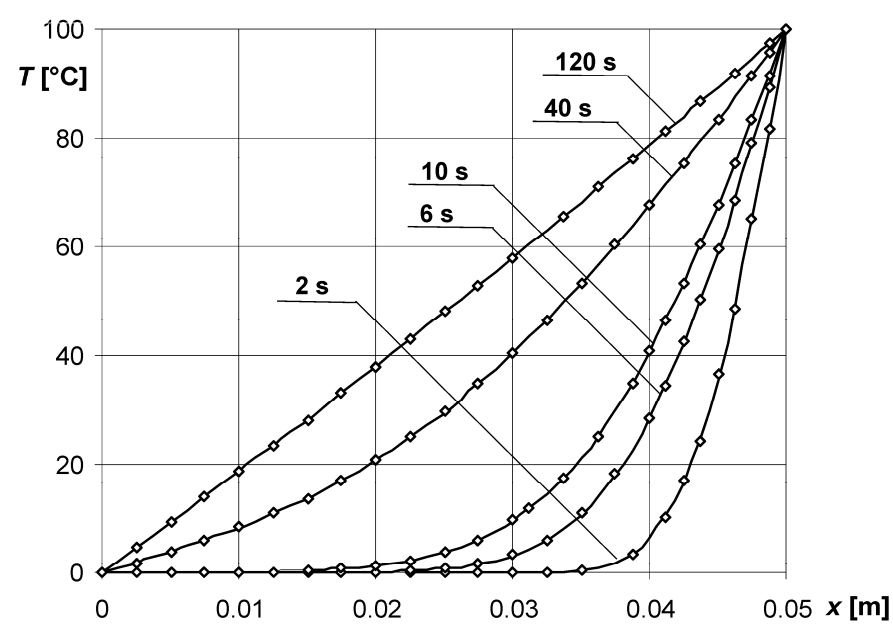

Fig. 3. LBM (symbols) and FDM (solid lines) solutions - example 1

Figure 4 illustrates the temperature distributions under the assumption that for $x=0: \quad T(0, t)=150^{\circ} \mathrm{C}$ (Dirichlet condition) and for $x=L:-\lambda \partial T(x, t) / \partial x=$ $=\alpha\left[T(x, t)-T_{a}\right]$ (Robin condition, $\left.\alpha=10 \mathrm{~W} /\left(\mathrm{m}^{2} \mathrm{~K}\right), T_{a}=20^{\circ} \mathrm{C}\right)$. 


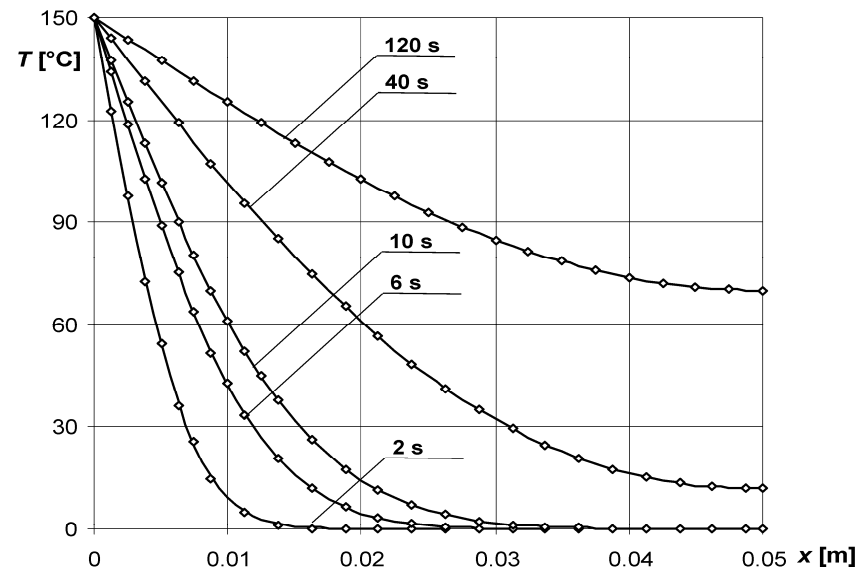

Fig. 4. LBM (symbols) and FDM (solid lines) solutions - example 2

\section{Conclusions}

The lattice Boltzmann method for the 1D Fourier equation supplemented by different boundary conditions and initial condition has been presented. The exemplary tasks have been solved both by the lattice Boltzmann method and by the explicit scheme of the finite different method. The good agreement of the solutions obtained has been observed.

\section{References}

[1] Mishra S.C., Mondal B., Kush T., Rama Krishna B.S., Solving transient heat conduction problems on uniform and non-uniform lattices using the lattice Boltzmann method, International Communications in Heat and Mass Transfer 2009, 36, 322-328.

[2] Mohamad A.A., Lattice Boltzmann Method Fundamentals and Engineering Applications with Computer Codes, Springer, London, Dordrecht, Heidelberg, New York 2011.

[3] Succi S., The Lattice Boltzmann Method for Fluid Dynamics and Beyond, Oxford University Press, New York 2001.

[4] Chaabane R., Askri F., Nasrallah S.B., Application of the lattice Boltzmann method for solving conduction problems with heat flux boundary condition, International Renewable Energy Congress, Sousse Tunisia 2009.

[5] Piasecka Belkhayat A., The interval lattice Boltzmann method for transient heat transport, Scientific Research of the Institute of Mathematics and Computer Science Czestochowa University of Technology 2009, 1(8), 155-160.

[6] Mochnacki B., Lara-Dziembek S., Weighted residua method as a tool of FDM algorithm construction, Scientific Research of the Institute of Mathematics and Computer Science Czestochowa University of Technology 2010, 1(9), 147-153.

[7] Majchrzak E., Mochnacki B., Metody numeryczne. Podstawy teoretyczne, aspekty praktyczne i algorytmy, Wyd. Politechniki Śląskiej, Gliwice 2004.

[8] Mochnacki B., Suchy J.S., Numerical Methods in Computations of Foundry Processes, PFTA, Cracow 1995. 